\title{
Bioprofiling of Cosmetics with Focus on Streamlined Coumarin Analysis
}

\author{
Constanze Stiefel, Tina Schubert, and Gertrud E. Morlock*(0)
}

Chair of Food Science, Institute of Nutritional Science, and Interdisciplinary Research Center (IFZ), Justus Liebig University Giessen, Heinrich-Buff-Ring 26-32, 35392 Giessen, Germany

\section{Supporting Information}

ABSTRACT: Facing the widespread use of cosmetic products in daily use and recognizing the very limited information obtained by target analysis, a method suited for comprehensive characterization of cosmetics was aimed at. The biological activity of ingredients of 20 cosmetics taken from 16 different product groups and their coumarin contents were investigated via chromatography linked to bioassays (direct bioautography) and mass spectrometry. It allows for screening a large number of cosmetic products within a short time to generate a more valid database on their coumarin content and their contribution to the overall exposure. Bioactivity profiling of cosmetics with regard to bioactive ingredients opens new avenues for a comprehensive characterization of important substances in products of daily use, helpful for the legally required safety and risk assessment of cosmetic products, especially for multiple product usage. As for coumarin, a ubiquitary fragrance compound of allergenic potential, which is under recurrent discussion due to its hepatoxic properties, it is necessary to be able to estimate the regular intake via cosmetics for a valid risk assessment. This newly developed bioprofiling method allowed a selective determination of coumarin down to $1.3 \mathrm{mg} \mathrm{kg}^{-1}$, even for very matrix-rich cosmetics despite minimalism in sample preparation. The declaration limits according to European Cosmetics Regulation were completely covered. Mean coumarin contents of 20 cosmetic products reached up to $2218 \mathrm{mg} \mathrm{kg}^{-1}$. The repeatabilities (\%RSD, $n=3$ ) were between 1.1 and $2.9 \%$, and the mean recoveries $(n=5)$ were between 96 and $102 \%$ for the different cosmetic matrices.

\section{INTRODUCTION}

Considering multiple product usage over a day, the overall impact of biologically active ingredients of cosmetics through the skin is a rough estimate. A screening method that more precisely informs on potential effects at a given rational effort is not existent and triggered this research. On the example of coumarin, the present challenge is illustrated in detail. Coumarin belongs to a chemical class of benzopyrones, which includes further naturally occurring derivatives, such as, umbelliferone (7-hydroxycoumarin), aesculetin (6,7-dihydroxycoumarin), or herniarin (7-methoxycoumarin), showing a wide variety of biological activities. As a main aroma compound of cinnamon (especially Cinnamomum cassia), coumarin is a natural ingredient of many food products. The odor-fixing properties and sweet, warm, vanilla-like scent of coumarin explain its use as a synthetic fragrance component or as a natural ingredient of various essential oils and plant extracts, such as sweet woodruff, tonka, or lavender, in a large number of cosmetic products. ${ }^{1,2}$

In the past, coumarin was repeatedly under discussion due to its potential toxicological properties. Species-specific animal testing data (e.g., of rats and dogs) indicated a possible hepatotoxicity after oral intake, ${ }^{3}$ whereupon the use of coumarin in its synthetic form was banned in the USA and numerous European countries since the mid-20th century. ${ }^{4,5}$ New scientific in vivo data ${ }^{6,7}$ let the Scientific Panel on Food Additives, Flavourings, Processing Aids and Materials in Contact with Food of the European Food Safety Authority (EFSA) allay the suspicion that coumarin may be genotoxic and may bind covalently to the DNA, which supports the induction of tumors. ${ }^{8}$ It is generally accepted that there are major interspecies differences regarding coumarin metabolism, which may be responsible for the diverse hepatoxicity data observed. $^{9-12}$ However, clinical data from therapeutic use of coumarin show that hepatotoxicity in man cannot completely be ruled out for sensitive individuals. ${ }^{13}$ Thus, it has to be supposed that a small group of humans may be susceptible to coumarin. Hence, the authority confirmed a hepatotoxic potential of coumarin. According to animal testing data, a noobserved-adverse-effect level for liver toxicity of $10 \mathrm{mg}$ coumarin $\mathrm{kg}^{-1}$ bw day ${ }^{-1}$ was determined for the most sensitive animal species (dog). Derived from this value and taking into account a safety factor of 100, the EFSA established a tolerable daily intake (TDI) of $0.1 \mathrm{mg}$ coumarin $\mathrm{kg}^{-1} \mathrm{bw} \mathrm{day}{ }^{-1}$. To

Received: May 6, 2017

Accepted: August 17, 2017

Published: August 31, 2017 
guarantee the safety of the consumers, the European Flavoring Regulation (EU) No. 1334/2008 laid down limit values between 5 and $50 \mathrm{mg} \mathrm{kg}{ }^{-1}$ for coumarin in cinnamoncontaining food, oriented to the TDI and the average daily intake of coumarin assuming a normal diet. These limits were also set under the proviso that coumarin is not added in its isolated or synthetic form but added as a natural part of a food ingredient like cinnamon powder. ${ }^{14}$

Scientific data show that coumarin penetrates easily and to a high proportion through the skin, i.e., up to 60 or even $100 \%$, depending on the used vehicle, $9,15,16$ and is therefore also systemically available. Inexplicably, in contrast to food, there is no maximum limit set by the European Cosmetics Regulation (EU) No $1223 / 2009$ for the usage of coumarin in cosmetics. ${ }^{17}$ Merely, due to the known sensitization potential of coumarin, ${ }^{18-20}$ the Cosmetics Regulation requires the declaration of coumarin in the ingredient list of cosmetics from a level of $0.01 \%$ (rinse-off products) and $0.001 \%$ (leave-on products). Additionally, the International Fragrance Association and the Research Institute for Fragrance Materials recommend specific limits for the usage of coumarin in different end products for the manufacturers in the perfume and cosmetic industry. ${ }^{21}$ These limits between $0.1 \%$ for lip products and $5 \%$ for rinse-off hair conditioners are based on a quantitative risk assessment, currently solely set to reduce the risk of sensitization against coumarin in the general population to a minimum.

Human exposure to coumarin via diet has been estimated to be around $0.02 \mathrm{mg} \mathrm{kg}^{-1} \mathrm{day}^{-1}{ }^{10}$ The exposure through the use of cosmetics is assumed to be about twice as high. ${ }^{10}$ Also, the German Federal Institute for Risk Assessment (BfR) calculated the dermal exposure to coumarin ${ }^{22}$ oriented at the average coumarin concentrations of different cosmetic products and the estimated daily exposure levels for different cosmetic product types, as published by the Scientific Committee on Consumer Safety (SCCS). ${ }^{23}$ Considering a correction factor for skin absorption, which was based on experimental data ${ }^{15}$ and assuming maximum intake levels (worst-case scenario), the BfR calculated an exposure level of $0.14 \mathrm{mg} \mathrm{kg}^{-1} \mathrm{bw} \mathrm{day}^{-1}$ for perfumes and $0.013 \mathrm{mg} \mathrm{kg}^{-1} \mathrm{bw} \mathrm{day}{ }^{-1}$ for body lotions or oils. As an average consumer normally uses multiple cosmetic products over the day, the overall coumarin concentration is expected to be even higher. Accordingly, for persons who use cosmetics regularly, the TDI can be significantly exceeded or at least partially reached. Therefore, the intake of coumarin via the dermal route should not be underestimated, especially as the metabolism of coumarin after oral administration or dermal application seems to be similar (no specific metabolism in the skin). ${ }^{9,10}$ However, the BfR pointed out that conclusive data about the coumarin concentration of different cosmetic products in the market are still low and further research is needed to make a final assessment.

To determine the concentration of coumarin in cosmetic products and to control the specified declaration requirements, both manufacturers and authorities need fast and reliable analytical methods. For the determination of coumarin in food and some plant extracts, different analytical methods are described. In early times, paper chromatography, thin-layer chromatography (TLC), and polarography were mainly used. ${ }^{24}$ Today, mostly high-performance liquid chromatography (HPLC) and gas chromatography (GC) are the methods of choice. $^{25-29}$ Many of these methods are selective and sensitive; however, they require relatively expensive instrumentation or a rather laborious sample preparation. A currently developed high-performance thin-layer chromatography (HPTLC) method for the determination of coumarin in different food products used postchromatographic derivatization with a methanolic potassium solution. ${ }^{30}$ Immersing the HPTLC plate in the alkaline solution led to an opening of the lactone ring of coumarin $^{31}$ and resulted in an intensive fluorescence (excitation wavelength at $360 \mathrm{~nm}$ ), which allowed a sensitive determination and quantification of coumarin in the food samples.

The present work was aimed at adapting the latter sensitive method to the examination of cosmetics, even those with a heavy matrix load. Hyphenation with various bioassay detection systems could allow a first insight regarding the bioactivity of different commonly used cosmetic ingredients. Direct bioautography has already been proven to be an effective tool for the identification of bioactive components in plant extracts, food, or waste water ${ }^{32-35}$ and may be just as useful for a first bioprofiling of cosmetic products. The targeted coupling with mass spectrometry (MS) may allow the characterization of active cosmetic ingredients of interest. Such a streamlined method will improve the knowledge on the extent of cosmetic usage, contributing to the total exposure of coumarin and further biologically active compounds.

\section{RESULTS AND DISCUSSION}

Facing the widespread use of cosmetic products in daily use while recognizing the very limited information obtained by target analysis, a method suited for comprehensive characterization of cosmetics was aimed at. The method focused on simplicity, rapidity, and high throughput screening of very different cosmetic products with different textures to increase the data set regarding the coumarin usage and biological effects of further ingredients.

2.1. Minimalism in Sample Preparation. After experiences with the preparation of food, ${ }^{30}$ ethanol seemed to be an effective solvent for cosmetic samples of a wide polarity range. However, for semisolid and especially solid formulations (e.g., lip care sticks), dispersibility of the samples was a limiting factor. Thus, a scent-free hand cream was spiked with a known amount of coumarin and extracted using ethyl acetate, acetonitrile, ethanol $\left(60{ }^{\circ} \mathrm{C}\right)$, and oxolane. Oxolane was preferred, as it showed an almost complete recovery of more than $98 \%$ for the hand cream (Figure S-1), while a sufficient dispersion or in most cases even a complete dissolution was reached. Compared with the other solvents, oxolane showed the best performance regarding both sample preparation and recovery for a wide range of product groups, such as alcoholbased deodorants, oil-in-water (o/w) emulsions, and (semi)solid products, such as lip care sticks or body butter. Because cosmetic products are mixtures of various substances and often include oils, waxes, or other fatty components, their incomplete solubility may be challenging for using a closed analytical system like HPLC with regard to clogging or contamination of the column. In the case of HPTLC, which is an open system that uses each separation phase only once, possible matrix contaminations are no issue. This allowed a minimum of sample preparation for all samples studied that was reduced to dissolution and, if required, membrane filtration.

2.2. Stability of Coumarin in Solution. The coumarin solution was stored at $6{ }^{\circ} \mathrm{C}$ in the dark. Ten aliquots, taken during 21 days, were analyzed for their coumarin content. Comparable concentrations of coumarin were obtained over the 3 week period (Figure S-2) with relative standard deviations (\%RSD, $n=10$ ) of 3.3 or $2.1 \%$, depending on the respective 


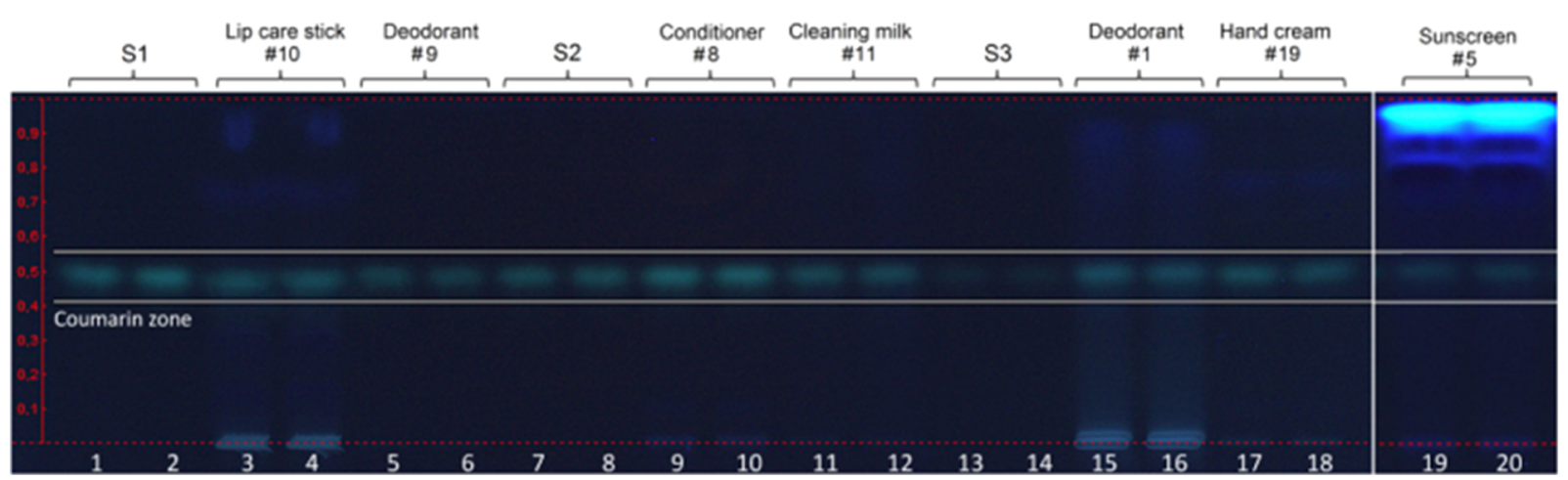

Figure 1. HPTLC chromatogram at UV $366 />400 \mathrm{~nm}$ of coumarin at $h R_{F} 50$ in different cosmetics (twofold overall analysis) with matrix mainly located at the start zone or in the front, as well as coumarin standard $\left(\mathrm{S} 1-\mathrm{S} 3,2.5-20 \mathrm{ng}^{\mathrm{band}} \mathrm{d}^{-1}\right)$.

application volume $(0.5$ or $2 \mu \mathrm{L}$ of the $10 \mathrm{ng} / \mu \mathrm{L}$ solution $)$. Thus, the stability of coumarin solutions for up to 3 weeks was proven.

2.3. Fluorescence Stability on the Plate. Postchromatographic derivatization with potassium hydroxide was chosen to visualize coumarin on the HPTLC plate. As the resulting fluorescence was light-sensitive, it was necessary to stabilize the fluorescent signal of the derivatized coumarin in the chromatogram. Both $30 \%$ paraffin in $n$-hexane and $10 \%$ methanolic poly(ethylene glycol) (PEG) solution were tested. However, PEG was advantageous regarding fluorescence stabilization and intensification (data not shown). The influence of light on the PEG-treated fluorescence was examined in detail over a period of $1 \mathrm{~h}$. Storage under daylight led to a strong decrease of the fluorescence of about $50 \%$ within $15 \mathrm{~min}$, whereas for storage in the dark, a small reduction in the fluorescence signal was observable in the first $10 \mathrm{~min}$, while the fluorescence was relatively stable over the next $30 \mathrm{~min}$ (Figure S-3). Consequently, the freshly derivatized chromatogram was instantly transferred to the TLC Scanner 4 and kept in the dark. After a waiting time of $10 \mathrm{~min}$, the measurement was performed in the fluorescence-stable period.

Immersion into the PEG solution did not only stabilize the fluorescent signal but also enhanced it, at least by a factor of 3 (Figure S-3). A standardized procedure for the derivatization was established, which included defined drying times before and in between the immersions. Sufficient drying was crucial to prevent spreading of the coumarin zone during immersion. In contrast, the immersion time and immersion speed had only minimal influence on the shape and sharpness of the coumarin zone. Drying of the plate was performed on a plate heater and not under a stream of warm air because the latter resulted in a reduced fluorescence signal, peak broadening, and worsened precision (data not shown).

2.4. Method Validation. For the newly developed method, selectivity, recovery, repeatability, reproducibility, limit of detection (LOD), and limit of quantitation (LOQ) were examined. As the derivatization was highly selective and sensitive, coumarin could be determined at very small amounts, even in very matrix-rich cosmetics without interference by other UV-absorbing components or impurities (Figure S-4). For recovery, three different cosmetic formulations (alcoholbased deodorant, o/w emulsion hand cream, and body oil) were spiked with two different coumarin amounts (50 and 100 $\mu \mathrm{g}$ per $0.4 \mathrm{~g}$ ) to represent a content found in commercially available cosmetic products. With oxolane as the extraction solvent, mean recovery rates between 96 and 102\% $(n=5)$ were determined for the three samples, confirming the applicability of the method for quite different cosmetic textures.

For repeatability of the coumarin result on the same plate (\% RSD, $n=3$, peak area) by threefold analysis of four different product samples (deodorant, body milk, body oil, and lip care stick), the standard deviations ranged between 1.1 and $2.9 \%$. Reproducibility (\%RSD, $n=2$ ) was between 0.7 and $3.7 \%$, determined on different days on different HPTLC plates for seven cosmetic samples with various textures (deodorant, body butter, cleaning milk, face cream, body lotion, bath essence, and conditioner). Including sample preparation, reproducibility (\% $\mathrm{RSD}, n=2$ ) was between 1.7 and $5.8 \%$ (overall analysis) for the 20 cosmetics. These results confirmed the good precision of the method.

LOQs for the coumarin determination were $500 \mathrm{pg}^{\text {band }}{ }^{-1}$ and $1.3 \mathrm{mg} \mathrm{kg}^{-1}$ for the samples. LOD was determined to be $200 \mathrm{pg} \mathrm{band}^{-1}$ for standard solutions (Figure S-5). The European Cosmetics Regulation prescribes a declaration of coumarin from a concentration of $0.01 \%$ for leave-on and $0.001 \%$ for rinse-off products. Thus, with an about 10 times lower LOQ the developed method is well-suited to prove the declaration requirements of coumarin in cosmetics.

The developed method showed a good linearity in the calibration range of $0.5-40 \mathrm{ng}^{\text {band }}{ }^{-1}$ with a relative standard deviation of the calibration function of $2.4 \%$ and a correlation coefficient of 0.9998 (Figure S-6). At higher amounts of coumarin on the plate, the fluorescence signal was too intense and the applied coumarin volumes started to spread slightly on the plate during application. This resulted in the formation of a double peak, noticed during fluorescence measurement. For routine analysis, a three-point calibration was applied.

2.5. Fast and Accurate Sample Analysis to Support Risk Assessment. The applicability of the method was proven for 20 cosmetic products of a wide formulation range, i.e., ethanol-based deodorants, o/w emulsions with light texture (e.g., body milk), semisolid cream formulations for hand and face, and body oils and solid products (lip care stick). Chromatography was performed on HPTLC plates, silica gel 60 , with a mixture of $n$-hexane, ethyl acetate, and ammonia $(3.8: 1.3: 0.05, \mathrm{v} / \mathrm{v} / \mathrm{v})$ up to a migration distance of $55 \mathrm{~mm} .^{30}$ The addition of that small volume of ammonia as a modifier resulted in an optimal sharpness of the coumarin band at $h R_{\mathrm{F}}$ $50 \pm 2$. Despite the minimal sample preparation, a baseline separation from matrix components was evident (Figure S-4) and a fast development time of less than 15 min was reached. Even in the case of the examined sunscreen sample with a high content of UV filters, which showed partly a strong 
fluorescence themselves, the UV filters were clearly separated from the coumarin band. This allowed a sensitive and selective quantification of coumarin (Figure 1, tracks 19 and 20).

As to be expected, there were big differences regarding the coumarin content, which varied between 1.3 and $2218 \mathrm{mg} \mathrm{kg}^{-1}$ (Table 1). The highest concentration was measured for a bath

Table 1. Overview of the Determined Coumarin Content of the Different Examined Cosmetic Samples (Twofold Overall Analysis Including Extraction)

\begin{tabular}{rlcc} 
no. & product type & $\begin{array}{c}\text { coumarin content } \pm \text { SD } \\
\left(\mathrm{mg} \mathrm{kg}^{-1}\right)\end{array}$ & $\begin{array}{c}\% \mathrm{RSD} \\
(\%, n=2)\end{array}$ \\
1 & deodorant 1 & $1.3 \pm 0.4$ & 2.7 \\
2 & body lotion 1 & $312.1 \pm 0.7$ & 2.2 \\
3 & deodorant 2 & $36.4 \pm 0.7$ & 1.9 \\
4 & face cream 1 & $4.5 \pm 0.2$ & 3.3 \\
5 & sunscreen & $26.6 \pm 1.4$ & 5.1 \\
6 & face cream 2 & $28.1 \pm 1.0$ & 3.5 \\
7 & bath additive 1 & $901.6 \pm 18.2$ & 2.0 \\
8 & conditioner & $28.9 \pm 1.1$ & 3.9 \\
9 & deodorant 3 & $11.3 \pm 0.6$ & 5.4 \\
10 & lip balm & $15.3 \pm 0.4$ & 2.6 \\
11 & cleansing milk & $15.6 \pm 0.6$ & 3.8 \\
12 & body oil & $28.6 \pm 1.5$ & 5.3 \\
13 & body lotion 2 & $55.5 \pm 2.2$ & 3.9 \\
14 & body butter & $6.66 \pm 0.3$ & 5.8 \\
15 & bath additive 2 & $53.4 \pm 1.3$ & 2.5 \\
16 & body lotion 3 & $55.7 \pm 0.9$ & 1.7 \\
17 & hand cream 1 & $9.2 \pm 0.5$ & 5.4 \\
18 & deodorant 4 & $49.7 \pm 0.9$ & 1.9 \\
19 & hand cream 2 & $22.0 \pm 1.7$ & 4.2 \\
20 & bath additive 3 & $2218.9 \pm 44.7$ & 2.0 \\
\hline
\end{tabular}

additive, for which the need for a further dilution was evident during application. However, also the body care products, which are applied in a high amount on a large skin surface, contained coumarin up to $312 \mathrm{mg} \mathrm{kg}^{-1}$.

According to the European Cosmetics Regulation, manufacturers of cosmetic products are required to assess every product prior to its market launch in terms of its safety for human health. In this context, toxicological properties of all ingredients and the common exposure conditions must be taken into account. Because it is known that an average consumer uses several cosmetics every day, also the amount of applied coumarin can add up so that the proportion of the total intake of coumarin via cosmetic usage should not be underestimated. The cumulative human exposure value for different cosmetic products is estimated to be $17.4 \mathrm{~g} \mathrm{day}^{-1}{ }^{23}$ Because coumarin shows a high dermal absorption value of $60-$ $100 \%$, the majority of the topically applied fragrance compounds is systemically available. ${ }^{10}$

To evaluate the safety of coumarin, it is useful to resort to the earlier mentioned TDI value of $0.1 \mathrm{mg} \mathrm{kg}^{-1} \mathrm{bw} \mathrm{day}{ }^{-1}$. It is generally agreed that cosmetics should exhaust the TDI to a maximum of $20 \%$. For example, the amounts of face or hand cream needed to reach $20 \%$ of the TDI are much higher than the daily exposure levels estimated by the SCCS (Table S-2). However, in the case of the analyzed body lotion, the required amount of $3.8 \mathrm{~g}$ product is clearly below the estimated daily use level of $7.8 \mathrm{~g}$. For an average adult, the TDI would be exhausted to over $40 \%$ by usage of the lotion if the SCCS assessment is considered. In the case of a child, the TDI would even be exceeded by over $60 \%$. Also, bath additives seem to have a high relevance in this context due to the relatively high coumarin content. However, a bath additive gets normally highly diluted by the bathing water during application, so an additional retention factor must be considered. Although only a part of the cosmetics in the market contain coumarin, it can be seen that a significant portion of the TDI value could possibly be exhausted alone by the regular application of certain products, especially for cosmetics applied on the whole body. If it is taken into account that an average consumer uses various cosmetic products during the day, the coumarin concentration is likely to increase. Accordingly, it can be confirmed that cosmetic products can contribute significantly to the overall exposure to coumarin.

2.6. HPTLC-MS Confirmation. Postchromatographic derivatization of coumarin is the first choice for routine screening of cosmetics due to its high sensitivity and selectivity as well as its simple and fast performance. However, targeted coupling with MS could also be used to confirm the results in uncertain cases. As mass spectra are recorded from the zones of interest only, intervals of MS cleaning were reduced due to the minimal matrix or background load. In a cosmetic, the HPTLC-MS full scan mass spectrum of the assigned coumarin zone showed the basepeak at $m / z 147[\mathrm{M}+\mathrm{H}]^{+}$and another pronounced signal at $m / z 169[\mathrm{M}+\mathrm{Na}]^{+}$(Figure 2). Furthermore, two signals at $\mathrm{m} / z 179$ and 201 indicated the addition of two hydroxyl groups. A hydroxylation of coumarin is conceivable due to the alkaline conditions. Sodium adducts are also observed, as sodium is ubiquitously present.

At a migration distance comparable to the coumarin zone, a plate background was recorded and subtracted from the analyte spectrum to reduce signals caused by the mobile phase, plate, or MS system. As the underivatized coumarin zones are both colorless and nonfluorescent, the outer track of the plate was cut off and derivatized to determine the correct migration distance for coumarin on the remaining plate. Positioning was facilitated on HPTLC plates with a fluorescence indicator because coumarin showed fluorescence quenching at concentrations $\geq 100 \mathrm{ng}$ band $^{-1}$ (Figure $3 \mathrm{~A}$ ). After the recording of mass spectra, the plate can be derivatized to confirm the proper positioning of the elution head on the zone of interest, as the oval elution head eluted not the complete substance zone but only about $60 \%$. Thus, the residual substance amount was suited for post-MS visualization. The single quadrupole MS determination showed a substantially poorer sensitivity with a LOD of $500 \mathrm{ng}$ band $^{-1}$ for sample and standard zones, when compared with fluorescence measurement. However, the use of high-resolution MS (HRMS) ${ }^{30}$ or MS-MS will improve the LOD.

2.7. HPTLC-DPPH ${ }^{\bullet}$ Assay. The $\mathrm{DPPH}^{\bullet}$ assay provides a simple way to evaluate the free-radical scavenging activity of a substance and thus its antioxidant potential. Compared with the sum parameter assay, which indicates the overall radicalscavenging activity of a sample, dipping of the HPTLC plate into the reaction solution allows an assignment to specified single ingredients. In addition, a first estimation can be made to what extent individual components contribute to the overall antioxidant activity of a sample. Cosmetic ingredients with a positive response were indicated as light white zones on a purple background. For coumarin, no antioxidant activity was observed even for high coumarin concentrations of up to 800 ng band ${ }^{-1}$ (Figure 3C), whereas the coumarin substance band was clearly seen at UV $254 \mathrm{~nm}$ (Figure 3A). However, light 


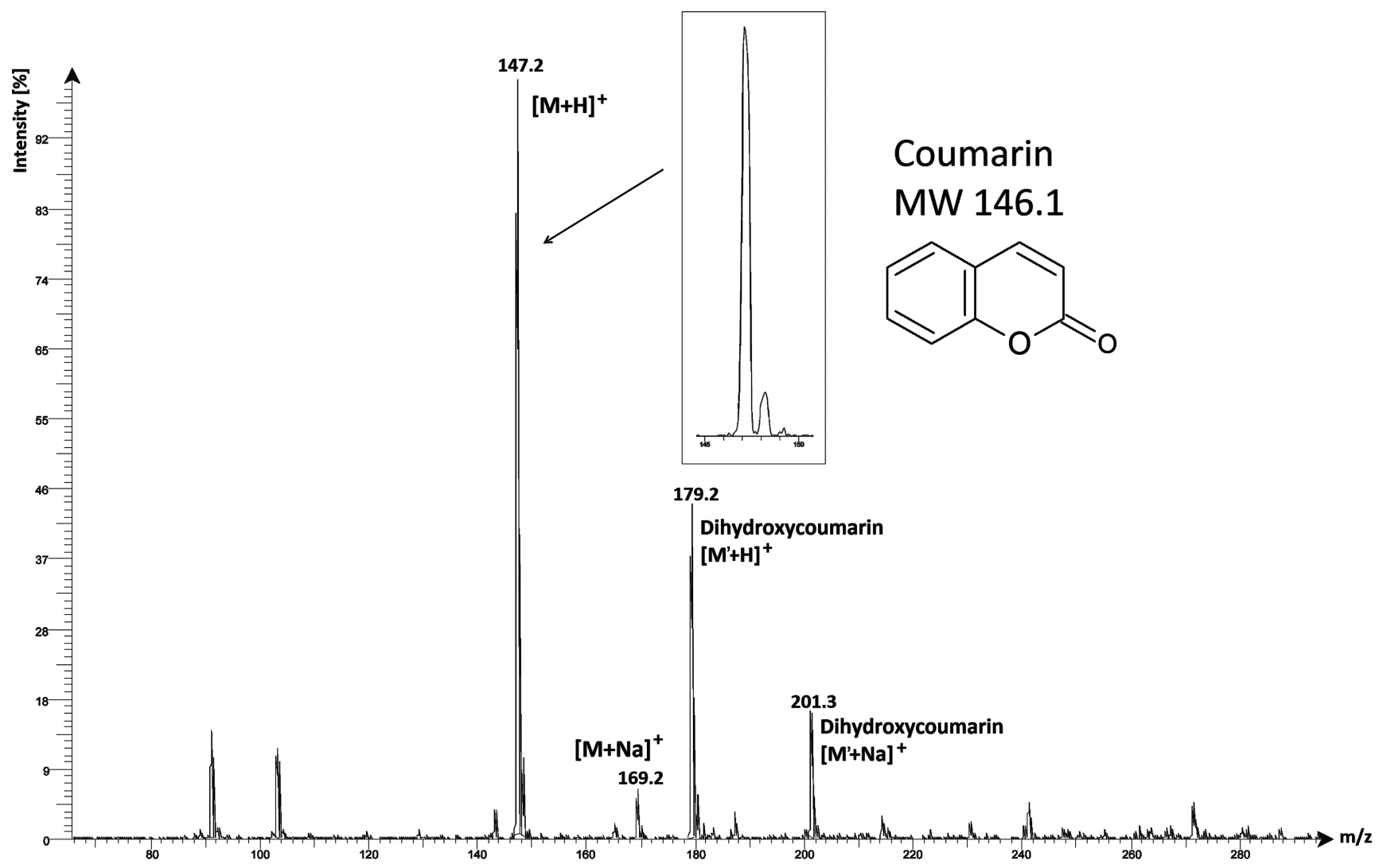

Figure 2. HPTLC-MS of a coumarin zone in a cosmetic sample showing as basepeak the protonated molecule at $m / z 147[\mathrm{M}+\mathrm{H}]^{+}$and its sodium adduct at $\mathrm{m} / z 169[\mathrm{M}+\mathrm{Na}]^{+}$as well as its respective dihydroxy forms at $\mathrm{m} / z 179$ and $\mathrm{m} / \mathrm{z} 201$.

substance bands were observed for nearly all cosmetic samples, indicating the antioxidative potential of some of the contained ingredients. This is not surprising because many cosmetics in the market advertise with a specific antioxidant potential of the product itself or some of its ingredients related to a possible protection function of those products for the skin. To gain more information about the ingredients that showed a strong free-radical scavenging activity during the assay, it is possible to characterize these substance zones by HPTLC-electrospray ionization mass spectrometry (ESI-MS). However, this was not the focus of this work. To generate more bioprofiling information regarding a potential activity of coumarin and the cosmetic samples, the chromatograms were subjected to further bioassays.

2.8. HPTLC-A. fischeri Bioassay. The bioluminescence of the Gram-negative $A$. fischeri bacteria can be influenced by bioactive compounds. Depending on the respective effect of a compound (positive or negative), lightened or dark zones are generated on a bluish luminescent background (depicted as a grayscale image). Coumarin concentrations of up to $800 \mathrm{ng}$ band $^{-1}$ generated a dark zone, indicating a negative impact on the bacterial growth (Figure 3D). Also lower concentrations generated a dark substance zone during the first minutes of imaging. However, the effect was not stable over the whole measurement time $(30 \mathrm{~min})$, which indicates an adaption or recovery of the bacteria after some minutes and therefore only a short-term effect. Also, the observed cosmetic samples showed various bioactive zones at different $h R_{\mathrm{F}}$ values, indicating some kind of bioactive potential of some of the contained ingredients (Figure 3D). Further investigation would be interesting to assign the bioactive substances to specific cosmetic ingredients by HPTLC-ESI-HRMS.

2.9. HPTLC-pYes Bioassay. Coumarin is not known to show a pronounced hormonal activity. However, cosmetics in general are repeatedly under discussion because of possible hormone-active ingredients. Therefore, the cosmetic samples were additionally examined for a possible endocrine activity of the contained ingredients. To generate sharply bounded zones, a recently developed workflow on water-wettable reversed phases was used. ${ }^{36}$ As to be expected, coumarin did not show a positive response. However, three cosmetic samples (Figure 3E, sunscreen no. 5; cleansing milk no. 11; body lotion 3 no. 16) showed a clear positive response during the pYES assay, indicated by up to four blue fluorescent substance bands at UV $366 \mathrm{~nm}$ at $h R_{\mathrm{F}}$ values between 40 and 65 . Also, for the positive control (C, $150 \mathrm{pg}$ band $^{-1}$ estradiol), a bright fluorescent zone was detectable, confirming the accuracy of the bioprofiling method. Because of the $h R_{\mathrm{F}}$ values of the fluorescent zones and the labeling of the ingredients, the fluorescent substance bands were assigned to the parabens contained as preservatives and known for endocrine activity. ${ }^{37}$ In our reversed-phase separation, methyl paraben can be found at the highest and butyl paraben at the lowest $h R_{\mathrm{F}}$ value. In a deodorant of a natural cosmetic manufacturer (no. 1, Table 1, Figure 3E), two slightly fluorescent zones at similar $h R_{\mathrm{F}}$ values as 48 and 53 were observed. A clear assignment to specified compounds of the ingredient declaration was not possible. It can only be assumed that the observed weak response may be attributed to natural ingredients of the used essential oils as it is known that for some essential oil components like anethol also endocrine activity was observed. ${ }^{38}$ 

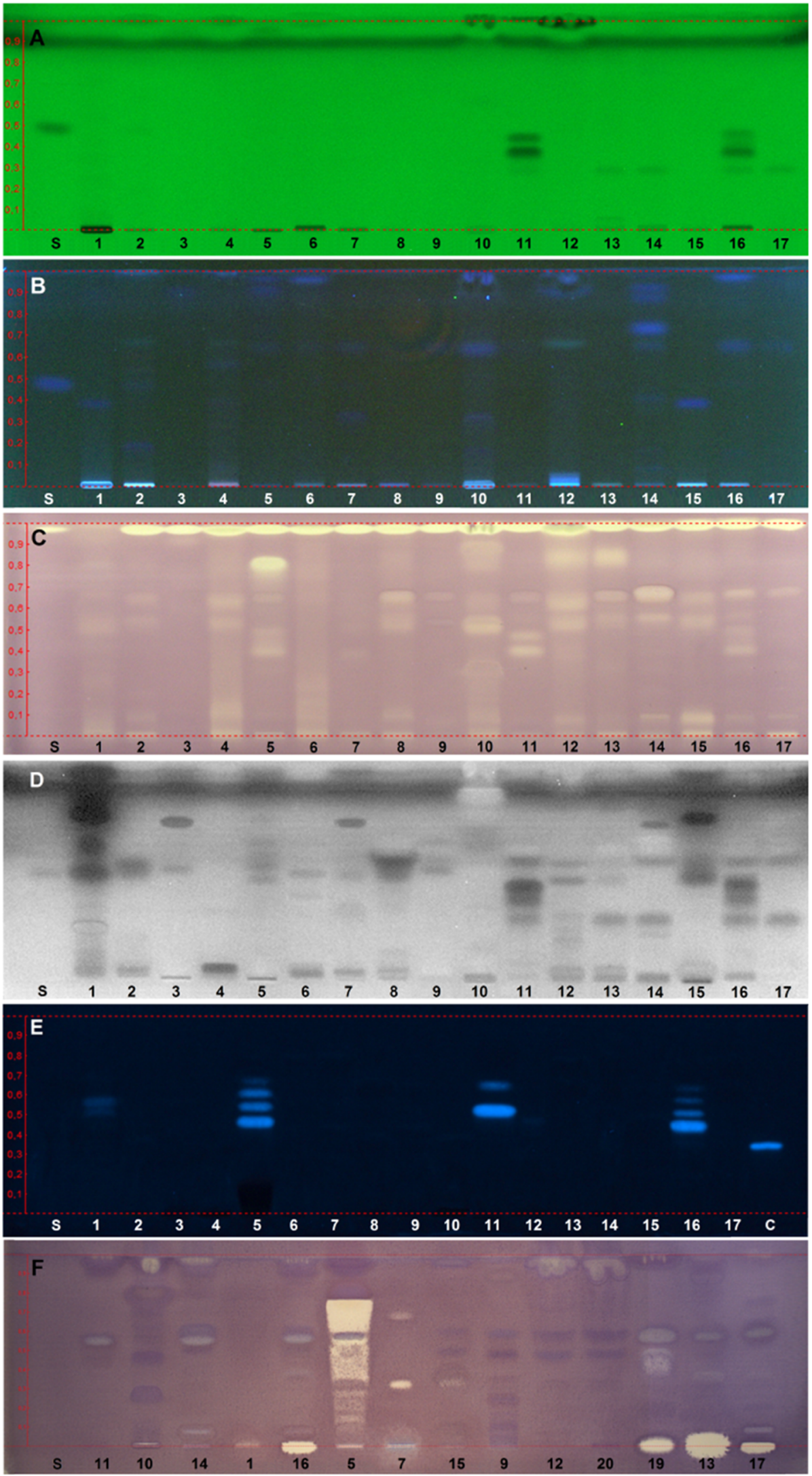

Figure 3. HPTLC-effect-directed analysis: chromatograms of different cosmetics (nos. 1-17 according to Table 1) and coumarin standard (S, 800 ng band ${ }^{-1}$ ) at (A) UV $254 \mathrm{~nm}$, (B) UV $366 \mathrm{~nm}$, and (C) after 2,2-diphenyl-1-picrylhydrazyl radical (DPPH ${ }^{\bullet}$ ) assay at white light illumination as well as bioautograms after (D) Aliivibrio fischeri bioassay as grayscale image, (E) pYES bioassay at UV $366 \mathrm{~nm}$ (estradiol $150 \mathrm{pg}^{\text {band }}{ }^{-1}$ as positive control C) and (F) Bacillus subtilis bioassay at white light illumination. 
2.10. HPTLC-B. subtilis bioassay. Gram-positive $B$. subtilis bacteria are used to discover potential antibiotic properties of a sample and its ingredients. The response is indicated as noncolored light zones on a deep purple background, attributed to an enzymatic reaction with 3-(4,5dimethylthiazol-2-yl)-2,5-diphenyltetrazolium bromide (MTT). In the B. subtilis bioassay, coumarin did not show any response, not even at as high amounts as $800 \mathrm{ng}$ band $^{-1}$, indicating no specific antibiotic potential (Figure 3F). However, the different cosmetic samples showed particularly intense antibiotic zones at the start zone of sample nos. 16, 19, 13, and 17, apart from various lightly colored antibiotic zones. Noteworthy with regard to an extreme antibiotic effect is the sunscreen sample no. 5, for which a large antibiotic area is visible along the separation, strongest in the upper $h R_{\mathrm{F}}$ range between 60 and 75. As many cosmetic products contain preservatives but also multifunctional ingredients, having combined properties like moisturizing and antimicrobial properties, it is not surprising that different samples show a positive response in the microbiological assays (Figure 3D,F). It is unclear to what extent such cosmetics with extreme antibiotic potential do impair the natural bacterial colonization on the skin and its protective function.

\section{CONCLUSIONS}

The bioprofiling of a wide variety of cosmetic products was developed and successfully demonstrated. The hyphenation of HPTLC with various bioassays gave information about the bioactivity of coumarin and other cosmetic ingredients, being helpful for the legally required safety assessment of cosmetic products. The method was also suited to screen a large number of cosmetic products within a short time for their coumarin content; 19 analyses in parallel took $55 \mathrm{~min}$ (application 10 min, chromatography $15 \mathrm{~min}$, derivatization and drying times $15 \mathrm{~min}$, and evaluation $15 \mathrm{~min}$ ). This corresponded to an overall analysis time of less than 3.5 min per sample. The solvent consumption was below $1 \mathrm{~mL}$ per sample. Although sample preparation was substantially reduced (mainly dissolution in oxolane) compared to that in other chromatographic methods, very good recovery rates, precisions, and detectabilities (LOQ of $1.3 \mathrm{mg} \mathrm{kg}^{-1}$ for even matrix-rich cosmetics) were obtained. Thus, this fast, sensitive, and simple HPTLC method was suited to generate a more valid database regarding the concentration of coumarin in cosmetics. These data may help in more precisely estimating to what extent the usage of cosmetics contributes to the overall coumarin exposure and may be used for further risk assessment, especially when multiple product usage is considered.

\section{EXPERIMENTAL SECTION}

4.1. Chemicals and Materials. Cosmetic products, for which coumarin was declared in the ingredients' list, were purchased from local drugstores or pharmacies. Coumarin $(\geq 99.0 \%)$ and 2,2-diphenyl-1-picrylhydrazyl radical (DPPH ${ }^{\bullet}$, 97\%) were obtained from Sigma-Aldrich (Steinheim, Germany). Potassium hydroxide $(>85 \%)$, oxolane $(\geq 99.5 \%)$, and methanol $(\geq 99.9 \%)$ were purchased from Carl Roth (Karlsruhe, Germany), and polyethylene glycol 400 was purchased from J.T. Baker Chemical Co. (Phillipsburg, NJ). Ammonia (25\%), paraffin (subliquidum), and $n$-hexane (>95\%) were obtained from Th. Geyer (Renningen, Germany). Reagents for the bioassays were referred to in the respective sections. HPTLC glass plates; silica gel 60, silica gel $60 \mathrm{~F}_{254}$, and RP-18 $\mathrm{WF}_{254}$ s (all $20 \times 10 \mathrm{~cm}^{2}$, layer thickness ca. 200 $\mu \mathrm{m})$; were delivered by Merck, Darmstadt, Germany. Prior to use, all plates were prewashed with a mixture of methanol and water $(4: 1, \mathrm{v} / \mathrm{v})$ up to a migration distance of $70 \mathrm{~mm}$ and dried at $110{ }^{\circ} \mathrm{C}$ for $10 \mathrm{~min}$.

4.2. Solutions. An aliquot of each cosmetic sample (400 $\mathrm{mg}$ ) was transferred to a $15 \mathrm{~mL}$ centrifuge tube and extracted in $5 \mathrm{~mL}$ oxolane in an ultrasonic bath for $5 \mathrm{~min}$. An aliquot of this solution was directly subjected to HPTLC or diluted appropriately with oxolane. Slightly cloudy suspensions were additionally centrifuged at $10000 \times g$ for $5 \mathrm{~min}$ and filtered through a $4.5 \mu \mathrm{m}$ membrane filter. The same sample was extracted twice and analyzed as a twofold determination. The stock solution of coumarin dissolved in oxolane $\left(5 \mathrm{mg} \mathrm{mL}^{-1}\right)$ was diluted 1:5000, 1:1000, 1:500, and 1:12.5 with oxolane (1, 5, 10, and $\left.400 \mathrm{ng} \mu \mathrm{L}^{-1}\right)$, for standard solutions $1,2,3$, and 4, respectively.

4.3. Application. Solutions were sprayed with Automatic TLC Sampler 4 (ATS4, CAMAG, Muttenz, Switzerland) as 6 $\mathrm{mm}$ bands ( $4 \mathrm{~mm}$ for HPTLC-MS) allowing 18 tracks to be applied on one HPTLC plate (distances from lower edge 8 $\mathrm{mm}$, from left side $15 \mathrm{~mm}$, and between bands $9.4 \mathrm{~mm}$ ), for a high sample throughput, even 19 tracks. For quantification, standard solution 2 was applied in volumes of $0.5,2.0$, and 4.0 $\mu \mathrm{L}\left(2.5-20 \mathrm{ng}\right.$ band $\left.^{-1}\right)$. In most cases, $4.0 \mu \mathrm{L}$ of each sample was applied twice on the plate. For linearity, limits of detection (LOD, S/N 3) and quantitation (LOQ $S / N$ 10), standard solutions 1 and 3 were applied twice ranging $0.2-3.0$ and 1.0$4.0 \mu \mathrm{L}$, respectively. For repeatability, the samples were applied threefold between 3.0 and $6.0 \mu \mathrm{L}$, and for reproducibility, between 1.0 and $6.0 \mu \mathrm{L}$. For recovery, the spiked products $(1.0$ $\mu \mathrm{L}$ each) were applied five times. For effect-directed detection, the samples ranged $2.0-5.0 \mu \mathrm{L}$ and coumarin solution 4 was $2.0 \mu \mathrm{L}$.

4.4. Chromatography and Derivatization. After $5 \mathrm{~min}$ chamber saturation, chromatography was performed on HPTLC plates, silica gel 60, with $n$-hexane, ethyl acetate, and ammonia (25\%) (3.8:1.3:0.05, v/v/v), in a twin trough chamber (CAMAG) up to a migration distance of $55 \mathrm{~mm}$ from the lower plate edge. The development time took about $12 \mathrm{~min}$. The chromatogram was dried on TLC Plate Heater III (CAMAG) at $50{ }^{\circ} \mathrm{C}$ for $5 \mathrm{~min}$. After cooling to room temperature, the plate was immersed into a $10 \%$ methanolic potassium hydroxide solution (immersion time $2 \mathrm{~s}$, immersion speed $2.5 \mathrm{~cm} \mathrm{~s}^{-1}$ ) and again dried on the plate heater for $5 \mathrm{~min}$. For fluorescence stabilization, the chromatogram was immersed into a $10 \%$ methanolic solution of polyethylene glycol (PEG) 400 and dried for $2 \mathrm{~min}$. Before scanning, the plate was stored for $10 \mathrm{~min}$ in the dark. For HPTLC-pYES, HPTLC plates RP$18 \mathrm{WF}_{254} \mathrm{~s}$ with a mixture of methanol and water $(7: 3, \mathrm{v} / \mathrm{v})$, were used.

4.5. Evaluation. Fluorescence measurement was performed at UV 366/>400 nm using TLC Scanner 3 (CAMAG, slit size 5 $\mathrm{mm} \times 0.3 \mathrm{~mm}$, scanning speed $20 \mathrm{~mm} \mathrm{~s}^{-1}$ ). The chromatogram was documented by a TLC Visualizer (CAMAG), captured by a Baumer Optronic DXA252 digital camera with a 12 bit per channel color depth charge-coupled device (CCD), $100 \mu \mathrm{m}$ spatial resolution, $1944 \times 952$ pixel and $1 \mathrm{~s}$ exposure time with a gain of 1 . Spot amplification was used to optimize the visual zone intensity. Data obtained was processed with winCATS 1.4.7.2018 or BioLuminizer 1.0.2.6107 (both CAMAG).

4.6. Method Validation. For recovery, $400 \mathrm{mg}$ of fragrance-free hand cream, deodorant, and body oil were 
spiked with 10 and $20 \mu \mathrm{L}$ of coumarin stock solution (fivefold determination each), vigorously vortexed $(2 \times 1 \mathrm{~min}$, VortexGenie 2, Scientific Industries, NY), diluted with $10 \mathrm{~mL}$ of oxolane, sonicated for $5 \mathrm{~min}$, and centrifuged at $10000 \times g$ for $5 \mathrm{~min}$. For LOQ calculation in a matrix-rich sample, $40 \mathrm{mg}$ of a fragrance-free hand cream was spiked with $10,20,40$, and 80 $\mu \mathrm{L}$ of coumarin solution 2 (corresponding to $0.0001-0.001 \%$ ), vigorously vortexed for $1 \mathrm{~min}$, stored for at least 1 day and dissolved in $1 \mathrm{~mL}$ of oxolane each.

4.7. HPTLC-MS. The elution head-based TLC-MS Interface (CAMAG, oval elution head $4 \times 2 \mathrm{~mm}^{2}$ ) was connected to the ESI interface of a single quadrupole mass spectrometer (ExpressIon CMS, Advion, Ithaca, NY). Underivatized chromatograms were used to avoid clogging of the system by salt deposits. The zones of interest were marked with a soft pencil (via comparison with a derivatized strip of the same plate) and eluted with methanol at a flow rate of $0.1 \mathrm{~mL}$ $\min ^{-1}$ using a HP 1100 ChemStation pump (Agilent, Waldbronn, Germany). Mass spectra were recorded in the full scan mode to obtain the total ion current chromatogram between $\mathrm{m} / z 50$ and 800 . The settings in the positive ionization mode were as follows: capillary voltage $150 \mathrm{~V}$, source voltage $10 \mathrm{~V}$, capillary temperature and source gas temperature $250{ }^{\circ} \mathrm{C}$, ESI voltage $4.5 \mathrm{kV}$, and detector offset 1250. A plate background was taken at a migration position comparable to the analyte zone (same migration distance) and was subtracted from the analyte spectrum. Data processing was performed using Mass Express 1.1.22.15 and Data Express 1.1.22.15 (both Advion).

4.8. HPTLC-DPPH` Assay. For radical-scavenging activity (antioxidant activity), the chromatogram was dipped into a 0.5 $\mathrm{mM}$ methanolic $\mathrm{DPPH}^{\bullet}$ solution $^{39}$ for $5 \mathrm{~s}$ using the TLC Chromatogram Immersion Device (CAMAG, immersion speed $\left.3.0 \mathrm{~cm} \mathrm{~s}^{-1}\right)$, dried in the dark at room temperature for $90 \mathrm{~s}$, heated at $60{ }^{\circ} \mathrm{C}$ on the TLC Plate Heater (CAMAG) for $30 \mathrm{~s}$, and documented with the TLC Visualizer (CAMAG) at white light illumination (reflectance mode).

4.9. HPTLC-A. fischeri Bioassay. According to Krüger et al., ${ }^{40}$ the dried chromatogram was immersed into the bacterial suspension (prepared according to DIN EN ISO 11348-1, part 1 , section 5) for $2 \mathrm{~s}$ at an immersion speed of $2 \mathrm{~cm} \mathrm{~s}^{-1}$ and placed into the Bioluminizer (CAMAG). Eleven luminescent plate images were captured with a cooled 16 bit high-resolution CCD camera (exposure time $50 \mathrm{~s}$ ) in time intervals of $3 \mathrm{~min}$ until $30 \mathrm{~min}$. To keep the plate moist during the image recording, the HPTLC plate compartment was humidified by a water-wetted filter paper (sized $15 \times 10 \mathrm{~cm}^{2}$ to cover the free space) and covered by a glass plate.

4.10. HPTLC-pYES Bioassay. According to Klingelhöfer et al., ${ }^{36}$ the dried chromatogram was automatically immersed into the yeast cell suspension (immersion speed $3.5 \mathrm{~cm} \mathrm{~s}^{-1}$, immersion time $5 \mathrm{~s}$ ), incubated horizontally under nearly $100 \%$ humidity at $30{ }^{\circ} \mathrm{C}$ for $3 \mathrm{~h}$, dried under a stream of cold air for 4 min, and immersed into a buffered methylumbelliferone galactoside solution $\left(0.5 \mathrm{mg} \mathrm{mL} \mathrm{m}^{-1}\right)$, followed by a second incubation $\left(1 \mathrm{~h}\right.$ at $\left.37^{\circ} \mathrm{C}\right)$. After immersion of the plate into an alkaline solution of glycine $(0.1 \mathrm{M}, \mathrm{pH} 12)$, the blue fluorescence of the generated methylumbelliferone was measured at 366/>400 nm using TLC Scanner 3 (CAMAG). Blue fluorescent zones indicated compounds with estrogenic activity documented at UV $366 \mathrm{~nm}$ (Reprostar 3 documentation system, CAMAG).
4.11. HPTLC-B. subtilis Bioassay. According to JamshidiAidji et al., ${ }^{41}$ the chromatogram was immersed into the bacterial suspension (immersion time $6 \mathrm{~s}$ and speed $3.5 \mathrm{~cm}$ $\mathrm{s}^{-1}$ ), incubated horizontally under nearly $100 \%$ humidity ( $2 \mathrm{~h}$ at $37{ }^{\circ} \mathrm{C}$ ) and immersed into a $0.2 \%$ phosphate-buffered salinebuffered MTT solution (immersion time $1 \mathrm{~s}$ and speed $3.5 \mathrm{~cm}$ $\mathrm{s}^{-1}$ ), followed by further incubation $\left(30 \mathrm{~min}\right.$ at $\left.37^{\circ} \mathrm{C}\right)$. The bioautogram was dried on the TLC plate heater (CAMAG) for 5 min at $50{ }^{\circ} \mathrm{C}$. Because of the reduction of MTT to formazan, bioactive zones were documented as bright white substance zones against a purple background with the TLC Visualizer (CAMAG) at white light illumination in the reflectance mode.

\section{ASSOCIATED CONTENT}

\section{Supporting Information}

The Supporting Information is available free of charge on the ACS Publications website at DOI: 10.1021/acsomega.7b00562.

Table S-1, estimation of the amount of cosmetics required to exhaust the TDI of coumarin to $20 \%$; Figure $\mathrm{S}-1$, coumarin stability in the solution stored at $6{ }^{\circ} \mathrm{C}$ in the dark over 21 days; Figure S-3, stability of coumarin fluorescence on the HPTLC plate, when derivatized solely with methanolic potassium hydroxide versus by additional stabilization with methanolic PEG solution; Figure S-4, proof of selectivity via densitogram overlay of the coumarin standard and four samples; Figure S-5, determination of the LOD via densitogram overlay of three standard tracks obtained by fluorescence measurement at 366/>400 nm; Figure S-6, linear calibration by fluorescence measurement at $366 />400 \mathrm{~nm}$ in the range between 0.5 and $40 \mathrm{ng}$ band $^{-1}$ (PDF)

\section{AUTHOR INFORMATION}

\section{Corresponding Author}

*E-mail: Gertrud.Morlock@uni-giessen.de. Tel: +49-641-9939141. Fax: +49-641-99-39149.

ORCID

Gertrud E. Morlock: 0000-0001-9406-0351

Notes

The authors declare no competing financial interest.

\section{ACKNOWLEDGMENTS}

We acknowledge Merck, Darmstadt, Germany, and CAMAG, Muttenz, Switzerland, for supply of plates and instrumentation.

\section{REFERENCES}

(1) Rastogi, S. C.; Johansen, J. D.; Frosch, P.; Menné, T.; Bruze, M.; Lepoittevin, J. P.; Dreier, B.; Andersen, K. E.; White, I. R. Contact Dermatitis 1998, 38, 29-35.

(2) Buckley, D. A. Br. J. Dermatol. 2007, 157, 295-300.

(3) Hazleton, L. W.; Murer, H. K.; Thiessen, R., Jr.; Tusing, T. W.; Zeitlin, B. R. J. Pharmacol. Exp. Ther. 1956, 118, 348-358.

(4) Cohen, A. J. Food Cosmet. Toxicol. 1979, 17, 277-289.

(5) Opdyke, D. L. Food Cosmet. Toxicol. 1974, 12, 385-388.

(6) Api, A. M. Food Chem. Toxicol. 2001, 39, 837-841.

(7) Swenberg, J. A. Covalent Binding Index Study on Coumarin; Report of Laboratory of Molecular Carcinogenesis and Mutagenesis; University of North Carolina: Chapel Hull, NC, April 2003, submitted by European Flavour and Flagrance Association (EFFA), Square Marie-Louise, 49, B-1000, Brussels.

(8) European Food Safety Authority (EFSA)EFSA J. 2004, 104, 136 EFSA-Q-2003-118. 10.2903/j.efsa.2004.104. 
(9) Ford, R. A.; Hawkins, D. R.; Mayo, B. C.; Api, A. M. Food Chem. Toxicol. 2001, 39, 153-162.

(10) Lake, B. G. Food Chem. Toxicol. 1999, 37, 423-453.

(11) Felter, S. P.; Vassallo, J. D.; Carlton, B. D.; Daston, G. P. Food Chem. Toxicol. 2006, 44, 462-475.

(12) Steensma, A.; Beamand, J. A.; Walters, D. G.; Price, R. J.; Lake,

B. G. Xenobiotica 1994, 24, 893-907.

(13) Abraham, K.; Wöhrlin, F.; Lindtner, O.; Heinemeyer, G.; Lampen, A. Mol. Nutr. Food Res. 2010, 54, 228-239.

(14) European CommissionOff. J. Eur. Union 2008, L 354/34.

(15) Yourick, J. J.; Bronaugh, R. L. J. Appl. Toxicol. 1997, 17, 153158.

(16) Beckley-Kartey, S. A.; Hotchkiss, S. A.; Capel, M. Toxicol. Appl. Pharmacol. 1997, 145, 34-42.

(17) European CommissionOff. J. Eur. Union 2009, L 342/59.

(18) Krautheim, A.; Uter, W.; Frosch, P.; Schnuch, A.; Geier, J. Contact Dermatitis 2010, 63, 262-269.

(19) Schnuch, A.; Uter, W.; Geier, J.; Lessmann, H.; Frosch, P. J. Contact Dermatitis 2007, 57, 1-10.

(20) van Oosten, E. J.; Schuttelaar, M. L.; Coenraads, P. J. Contact Dermatitis 2009, 61, 217-223.

(21) International Fragrance Association. IFRA Standard - 48th Amendment, 2015.

(22) Bundesinstitut für Risikobewertung. BfR Statement No 049/ 2007.

(23) Scientific Committee on Consumer Safety. SCCS/1564/15.

(24) Bogan, D. P.; Keating, G. J.; Reinhartz, H.; Duffy, C. F.; Smyth, M. R.; O'Kennedy, R.; Thornes, R. D. Analysis of Coumarins. Coumarins: Biology, Application and Mode of Action; O'Kennedy, R., Thornes, R. D., Eds.; John Wiley \& Sons: Chichester, 1997; pp 267302.

(25) Sproll, C.; Ruge, W.; Andlauer, C.; Godelmann, R.; Lachenmeier, D. W. Food Chem. 2008, 109, 462-469.

(26) Raters, M.; Mattisek, R. Eur. Food Res. Technol. 2008, 227, 637642.

(27) Jagerdeo, E.; Passetti, E.; Dugar, S. M. J. AOAC Int. 2000, 83, 237-240.

(28) Rastogi, S. C. J. Sep. Sci. 1995, 18, 653-658.

(29) Ren, Z.; Nie, B.; Liu, T.; Yuan, F.; Feng, F.; Zhang, Y.; Zhou, W.; Xu, X.; Yao, M.; Zhang, F. Molecules 2016, 21, 1511.

(30) Krüger, S.; Winheim, L.; Morlock, G. E. Food. Chem. 2018, 239, $1182-1191$.

(31) Haskins, F. A.; Gorz, H. J. Agron. J. 1957, 49, 493-497.

(32) Klingelhöfer, I.; Morlock, G. E. Anal. Chem. 2015, 87, 1109811104 .

(33) Jamshidi-Aidji, M.; Morlock, G. E. Anal. Chem. 2016, 88, 10979-10986.

(34) Morlock, G. E. ACS Symp. Ser. 2013, 1185, 101-121.

(35) Morlock, G. E.; Klingelhöfer, I. Anal. Chem. 2014, 86, 82898295.

(36) Klingelhöfer, I.; Morlock, G. E. J. Chromatogr. A 2014, 1360, 288-295.

(37) Golden, R.; Gandy, J.; Vollmer, G. Crit. Rev. Toxicol. 2005, 35, $435-458$.

(38) Tabanca, N.; Khan, S. I.; Bedir, E.; Annavarapu, S.; Willett, K.; Khan, I. A.; Kirimer, N.; Baser, K. H. Planta Med. 2004, 70, 728-735.

(39) Pozharitskaya, O. N.; Ivanova, S. A.; Shikov, A. N.; Makarov, V. G. Phytochem. Anal. 2008, 19, 236-243.

(40) Krüger, S.; Urmann, O.; Morlock, G. E. J. Chromatogr. A 2013, 1289, 105-118.

(41) Jamshidi-Aidji, M.; Morlock, G. E. J. Chromatogr. A 2015, 1420, 110-118. 